\title{
Lectores en ruta: Hans Christian Andersen por España. Una propuesta didáctica para un club de lectura
}

\author{
Readers on the road: Hans Christian Andersen in Spain. \\ A didactic proposal for a reading club
}

María Encarnación Carrillo García ${ }^{1}$

Como citar este artículo: Carrillo García, M.E. (2021). Lectores en ruta: Hans Christian Andersen por España. Una propuesta didáctica para un club de lectura, Pangeas. Revista Interdisciplinar de Ecocrítica (núm. 3) 61-68. https://doi.org/10.14198/PANGEAS.19139

\begin{abstract}
Resumen
En este estudio se realiza, en primer lugar, una revisión de las características tradicionales de un club de lectura, y se termina realizando una propuesta de evento letrado en la que se unirán el acto de leer y el acto de viajar, que hemos denominado, en líneas generales, “Lectores en ruta” y, de manera específica, “Lectores en ruta: Hans Christian Andersen por España" para ejemplificarlo a partir de una obra determinada. Completamos nuestra aportación con una propuesta de lecturas para cada una de las regiones de la geografía española que puede servir de inspiración para el diseño y la puesta en marcha de otras propuestas de “Lectores en ruta".
\end{abstract}

Palabras clave: club de lectura; evento letrado; nuevas literacidades; lectura; viaje.

\section{Abstract}

In this study, we carried out a first review of the traditional characteristics of a book club, and we ended up making a proposal for a literary event in which the act of reading and the act of traveling, which we have called: "Readers on the road" and, more specifically, "Readers on the road: Hans Christian Andersen in Spain" in order to exemplify it from a specific work. We complete our contribution with a proposal of readings for each of the regions in the Spanish geography, which can serve as inspiration for the design and implementation of other proposals regarding "Readers on the road".

Key words: book club; literate event; new literacies; reading; travel.

1. María Encarnación Carrillo García. Universidad de Murcia. mariaencarnacion.carrillo@um.es https://orcid.org/0000-0002-8741-8412 


\section{INTRODUCCIÓN}

El célebre escritor danés Hans Christian Andersen, gran autor de cuentos infantiles, fue uno de los viajeros decimonónicos que viajaron por España, amantes de la imagen romántica que este país tenía en aquellos tiempos. Fruto de aquella experiencia trascurrida en 1862, Andersen escribió Viaje por España, una obra en la que nos relata su periplo por numerosas ciudades de la geografía española.

A partir de la lectura de este texto, proponemos el taller de lectura "Lectores en Ruta", un evento letrado, en el que la lectura y el viaje serán concebidos en su conjunto como una actividad cultural relacionada con el modo de lectura que se plantea en este tipo de taller. Lectores en Ruta es una propuesta de taller de lectura a partir de un libro de viajes donde los lectores se convierten en lectores de viajes, que además interactúan con el medio geográfico - de manera virtual y física- que describe el libro.

Para ilustrar esta propuesta partiremos del episodio descrito por Andersen a su paso por Murcia y Cartagena, y propondremos prácticas de lectura relacionadas con los lugares por los cuales transitó el escritor. Con nuestra aportación queremos reflexionar acerca de las nuevas literacidades, en relación a las cartografías lectoras existentes, y reflexionaremos sobre los club de lectura y las posibilidades educativas, culturales y de ocio del proyecto "Lectores en ruta" que proponemos.

\section{CLUBES DE LECTURA:}

\section{PRÁCTICA SOCIAL Y CULTURAL CONTEXTUALIZADA}

Según diversos autores (Calvo, 2002; Candamio, 2003; De la Cruz y Saurín-Parra, 2005; Domingo, 2007), y a modo de resumen, diremos que un club de lectura está formado por un grupo de personas que leen al mismo tiempo un libro. Cada uno lo hace en su casa pero una vez a la semana, en un día y a una hora fijados, se reúne el grupo para comentar las páginas avanzadas desde el encuentro anterior; una actividad lectora que permite realizar por un lado una lectura personal e íntima y por otro brinda la posibilidad a los usuarios del mismo de compartir esa lectura con otras personas. Entre las diferentes modalidades que podemos encontrar destacan las que atenderán al tipo de lectura que realicen (narrativa, biografía, ciencia ficción, etc..), la lengua utilizada en la lectura (lengua materna, extranjera, primera o segunda lengua...), el tipo de usuarios (mujeres, ancianos, niños, presos...) y los objetivos perseguidos (educativos, u orientados al ocio). En ellos se dan dos roles: el lector o lectora y el coordinador o coordinadora. $Y$ el recurso utilizado es el libro objeto de lectura, que puede ser obtenido para su lectura por compra o por préstamo en alguna biblioteca pública o de centro docente. En lo que se refiere a la selección de las obras, las personas que realicen la labor de selección entre las cuales estará el coordinador, tendrán que haber leído el libro, hacer una selección en la que se incluyan clásicos de la literatura y novedades que tengan suficientes méritos literarios, y si la obra cuenta con una versión cinematográfica, a veces, se suele ver la película para comparar la adaptación de la obra al cine.

Como vemos, en los clubes de lectura la experiencia lectora participa de la lectura en soledad y de la lectura en un contexto social determinado; al respecto Arellano (1995) explica que uno de los espacios por excelencia donde tendrán acogida los clubes de lectores será la biblioteca, la cual será una alternativa atractiva y socializadora a la soledad del lector.

Domingo (2004) coincide con los autores arriba señalados en las características generales de los clubes de lectura y describe además, de forma detallada, los clubes de lectura de la ciudad de Barcelona hasta el 2004 según la lectura que realizan en ellos, entre los cuales encontramos verdaderos ejemplos de especialización como son los clubes relacionados con temas variados, en concreto:

- Inglés: se puso en funcionamiento en la Biblioteca Francesca Bonnemaison para complementar su servicio de autoaprendizaje de idiomas.

Actualmente se realizan colaboraciones puntuales con el British Council.

\footnotetext{
- Alemán: con el objetivo de dar a conocer el fondo de novelas en esta lengua, la Biblioteca Miquel Llongueras, con
} 
la colaboración del Goethe-Institut de Barcelona, puso en marcha este club, muy bien acogido por parte de los usuarios interesados en mejorar la conversación en esta lengua.

- Italiano: persiguiendo el mismo objetivo que el caso anterior, la Biblioteca Miquel Llongueras ha iniciado, en este curso, este club, con la colaboración del Instituto Italiano de Cultura de Barcelona.

- Novela histórica: en la Biblioteca Collserola-Josep Miracle.

- Literatura de viajes: puesto en marcha en la Biblioteca Fort Pienc. Cada sesión está dedicada a una zona geográfica concreta, ya sea un país o una ciudad.

- Teatro: con la colaboración del Teatre Nacional de Catalunya-TNC, se comentan obras tanto clásicas como contemporáneas en la Biblioteca Vila de Gràcia. Se suelen acompañar las sesiones con la asistencia de las personas inscritas a la representación pertinente en el TNC.

- Poesía: de acuerdo con su especialización, la Biblioteca Mercè Rodoreda invita a los usuarios a comentar poesía contemporánea catalana, castellana e hispanoamericana. Las sesiones están moderadas por el presidente de la Aula de Poesía de Barcelona.

- Novela barcelonesa: Ia Biblioteca Juan Marsé, cuya especialización es la novela barcelonesa contemporánea, acoge este club y lo complementa con un ciclo de charlas paralelas con la asistencia del autor de la novela.

- Novela negra: la Biblioteca Montbau, con la colaboración de Grup 62, aprovechó la revalorización de este género para organizar este club. Coincide con su propio centro de interés, y suele programar el pase de la película siempre que la haya.

- Novela latinoamericana: teniendo en cuenta la gran cantidad de usuarios potenciales latinoamericanos que se encuentran en su distrito, la Biblioteca Nou Barris abrió este club con la finalidad de dar a conocer la tradición literaria de esta procedencia.

- Cine y literatura: la Biblioteca Xavier Benguerel, especializada en cine, organiza este club, donde aparte de proyectar una película, se comenta la novela originaria y se reproduce su banda sonora original. Colaboran también con Grup 62. La Biblioteca de Roquetes ofrece así mismo otro club parecido a este con la colaboración de Projectart.

- Cómic: la Biblioteca Ignasi IglesiasCan Fabra, colaborando con Planeta DeAgostini hace un recorrido por autores, géneros y tipologías diversas: europeo, manga, americano, español, latinoamericano... Coincide también con su propia especialización.

- Juvenil: con la finalidad de fomentar la lectura entre los jóvenes, las bibliotecas Montbau, Nou Barris, Clarà, Sant Pau-Sta. Creu i Ignasi Iglesias-Can Fabra organizan estas sesiones de lectura de forma similar.

Por otro lado, en cuanto a su funcionalidad social, Cortés (2011) explica que los clubes de lectura son un claro ejemplo de cultura democrática puesto que se convierte en un instrumento capaz de favorecer el espíritu crítico de la sociedad a través de actividades como la comparación y el análisis.

También encontramos ejemplos de clubes de lectura con carácter terapéutico, clubes que mantienen el componente social, cultural y de disfrute, como apuntan Castillo y Barra (2005) en la descripción de la experiencia de un club de lectura realizado con personas con discapacidad psíquica; un club que obtuvo buenos resultados según las valoraciones de los participantes, los responsables y los supervisores.

Tal y como acabamos de exponer, podemos decir que los clubes de lectura son actividades sociales y culturales de diversa índole, que giran 
en torno al acto individual de leer un libro y al acto social de dialogar sobre dicha lectura.

En la actualidad, y debido en gran parte a la situación de confinamiento que a nivel mundial se está viviendo por la pandemia originada por la Covid-19, están proliferando otras formas de clubes de lectura en formato online, en diferentes plataformas digitales de videoconferencia como por ejemplo Zoom o Google Meet, entre otras. Además existen aplicaciones que facilitan la reunión de los clubes de lectura a nivel mundial, como es Meetup (www.meetup.com), por poner un ejemplo, donde se puede asistir a las reuniones de clubes de lectura en diferentes países alrededor del mundo, y que están organizados por personas particulares, y que en numerosas ocasiones son gratuitos y públicos.

\section{NUEVAS LITERACIDADES Y EVENTOS LETRADOS}

Tal y como hemos comprobado en el apartado anterior, los clubes de lectura especializados son numerosos y atienden a objetivos diversos, y configuran lo que Martos (2010) denomina "cartografías lectoras de una ciudad", que según su definición, están formadas por los eventos de lectura y escritura que se dan en un lugar determinado, pudiéndose incluso localizar en un mapa, en la medida que se quiera investigar y registrar su prevalencia en el espacio y en el tiempo.

En lo que se refiere a la denominación de estos eventos letrados, Martos (2010) diferencia entre los eventos letrados convencionales que suelen inspirarse en formas más tradicionales de la cultura clásica, de forma asimétrica, donde los participantes tienen roles rígidos, como puede ocurrir en una conferencia magistral en la que uno habla y los demás escuchan; frente a una propuesta de evento letrado que potencie la participación, la horizontalidad de la comunicación y la movilidad de roles. $Y$ a este respecto explica que en ellos deben tomar protagonismo la participación del lector, la narración colectiva o el juego como principio de exploración.

\section{PROPUESTA PARA UN CLUB DE LECTURA: LECTORES EN RUTA}

A continuación, inspirándonos en las premisas apuntadas por Martos (2010) sobre los nuevos eventos letrados vamos a desarrollar nuestra propuesta de club de lectura centrada en dos parámetros principales: la lectura y el viaje; que confluirán ambos en el acto social final.

En concreto proponemos el evento letrado "Lectores en ruta", una propuesta multidisciplinar que parte en primer lugar de un libro determinado que se desarrolle en un espacio físico y geográfico concreto localizable en un mapa, para seguir en segundo lugar con la lectura individual de la obra, ideando posteriormente un ejercicio de georreferenciación (García, 2018) de las geografías literarias en un mapa, terminando con el acto social grupal final que consistirá en realizar un viaje, excursión o visita - dependiendo de la cercanía o lejanía del lugara los escenarios del libro. Con una organización grupal democrática donde los miembros del grupo contribuirán de forma consensuada en la organización de las lecturas que se han de realizar y en la dinamización de las sesiones grupales en el espacio de reunión utilizado, que podría ser un centro social, una biblioteca, un centro educativo, por poner algunos ejemplos; $y$, finalmente, contribuyendo en la preparación de las visitas guiadas de cada ciudad.

\section{HANS CHRISTIAN ANDERSEN EN RUTA POR ESPAÑA}

Para ejemplificar nuestra propuesta de lectura, proponemos un evento letrado que se desarrollará a partir de la obra Viaje por España de Hans Christian Andersen (2004).

El exotismo, las costumbres y las particularidades del carácter español atrajeron a un gran número de escritores y pensadores del siglo XIX, principalmente europeos, que se lanzaron a recorrer nuestro país y volcaron sus experiencias en distintos libros de viajes. Entre otros viajeros ilustres de aquellos tiempos, cabe destacar a Alejandro Dumas, Washington Irving, Teófilo Gautier, Victor Hugo o el autor que nos 
ocupa, Hans Christian Andersen. El escritor danés realizó, entre septiembre y diciembre de 1862 , un viaje por varias regiones españolas cuyas impresiones quedaron recogidas en la obra publicada en Dinamarca al año siguiente con el título I Spanien. Este viaje supuso para Andersen hacer realidad su viejo deseo de conocer nuestro país; un sueño que se había forjado cuando era niño, mientras jugaba con los soldados españoles destacados en Odense durante las guerras napoleónicas. Desde entonces España formaba parte de su imaginario, el cual ya había vertido en algunas de sus obras anteriores a su visita.

Este libro constituye un valioso testimonio acerca de las costumbres y la vida cotidiana en España decimonónica. La plasticidad de sus descripciones, la agilidad de su prosa y la agudeza mostrada para destacar entre la abigarrada realidad el detalle revelador o el tipo característico, hacen de esta obra un valioso texto literario y un documento histórico de primer orden. Un texto que permitirá, a su vez, hacer una reflexión ecocrítica sobre la evolución de los espacios descritos en la obra en comparación con su estado en la actualidad.

\section{LA PROPUESTA DIDÁCTICA}

\section{"Lectores en ruta: Viaje por España de Hans Christian Andersen"}

Podemos decir que el evento letrado que proponemos es una actividad cultural que tiende al movimiento y que invita a sacar de las páginas del libro las experiencias vividas por los personajes en los lugares por los que transcurre la acción, convirtiendo el acto de leer, también, en un acto social y dinámico, en el que el libro marcará la ruta de viaje a realizar, el camino a seguir por los "Lectores en ruta" participantes.

Este proyecto de club de lectura tiene los siguientes objetivos:

- Poner en marcha diferentes clubes de lectura en los lugares que visitó Andersen. Estos clubes de lectura pueden realizarse en diferentes contextos: educativos y culturales formales e informales; y se pueden celebrar en centros educativos, centros culturales, asociaciones y bibliotecas, por poner algunos ejemplos. Cada club de lectura debe planificarse por una persona que se encargue de la coordinación.

- Leer la obra Viaje por España de Hans Christian Andersen.

- Planificar un evento letrado en cada una de las ciudades que describe el libro que consistirá en: cartografiar los lugares descritos en la obra, para lo cual se pueden utilizar aplicaciones digitales como Tourist Eye, Google Map, o Geolocalizar, por ejemplo; y preparar los pasajes del libro relacionados con cada espacio geográfico. Cada club de lectura se encargará de realizar el de su ciudad.

- Realizar los encuentros de los clubes de lectura por toda la geografía española descrita en la obra, donde los clubes anfitriones guiarán a los clubes visitantes por su ciudad y realizarán la lectura dramatizada de los pasajes literarios del lugar. En esta actividad, cada club de lectura se encarga de gestionar su viaje en lo referente a desplazamiento, hotel y manutención. Para ello los clubes de lectura se dividirán en club de lectura anfitrión y clubes de lectura visitantes.

- Los clubes de lectura se podrán organizar en las ciudades que el libro describe; siguiendo el orden de los capítulos del libro y que se atienen al orden en el que fueron visitadas, las ciudades son: Barcelona, Valencia, Almansa, Alicante, Elche, Murcia, Cartagena, Málaga, Granada, Gibraltar, Ceuta, Cádiz, Sevilla, Córdoba, Madrid, Toledo, Burgos, Vitoria, San Sebastián e Irún.

- Al finalizar, realizar una exposición con los documentos generados por los clubes de lectura: mapas, lecturas, fotografías y vídeos de las zonas realizadas por los clubes anfitriones y visitantes. Esta exposición puede ser en formato físico, para ser expuesta en un lugar determinado a elegir, y que puede ser itinerante entre 
las diferentes ciudades participantes. También existe la posibilidad de realizar una exposición virtual de las visitas, y para lo cual se puede elegir diferentes formatos digitales: blogs, webs, muros digitales (www.padlet.com), entre otros formatos.

A continuación exponemos un ejemplo de las actividades que se derivan de la obra expuestas anteriormente en lo que se refiere a la visita que realizó Andersen a la Región de Murcia, en concreto, a las ciudades de Murcia y Cartagena. Las actividades que el club de lectura de Murcia serán las siguientes:

- Lectura de la obra Viaje por España de Hans Christian Andersen.

- Cartografiar las zonas que describe en las ciudades de Murcia y Cartagena. Esta cartografía se puede hacer de diferentes maneras, utilizando mapas de las dos ciudades en los cuales se localizan los lugares. Para ello se pueden utilizar aplicaciones digitales que faciliten la georreferenciación.

- Los lugares que se han de georreferenciar en Murcia, según la obra, serán: la Glorieta, la Catedral, la calle San Leandro, la Plaza de los Apóstoles, los Jardines del Malecón y el río Segura (en el libro: el arrabal), el Barrio del Carmen (en el libro: la Alameda), la calle de los Caballeros y el casino.

\section{- Los lugares a georreferenciar en} Cartagena, según la obra, serán: Casa Spotturno (la casa del Cónsul danés en esa época), el puerto marítimo, el Fuerte de Navidad, el Castillo de Santa Bárbara, Sierra Minera-La Unión (en el libro: minas de plata), Estación de ferrocarril (en el libro: estación que uniría Cartagena con Madrid y que estaba en obras), los astilleros, la dársena, y el casino.

- Preparar los pasajes literarios relacionados con cada una de las localizaciones para ser leídos por el club de lectura murciano durante la visita a los lugares descritos en el libro, y a cuya visita se podrán unir los diferentes clubes llegados de toda la geografía española, y para los cuales el club anfitrión hará de guía por las dos ciudades trabajadas: Murcia y Cartagena.

- Realizar una exposición virtual en un blog del club de lectura con los mapas realizados, las lecturas seleccionadas, y los vídeos y fotografías de las visitas.

Es interesante destacar que, a veces, el viaje relacionado con la visita de las geografías literarias de las obras pudiera no realizarse por motivos diversos, como es el caso actual, en la que la movilidad de los ciudadanos se ha visto restringida debido a la situación de confinamiento derivada de la pandemia; debido a ello, los coordinadores de los clubes de lectura podrían plantear la posibilidad de realizar el club de lectura de forma virtual por completo, con encuentros virtuales para hablar sobre la obra, y con sesiones de videoconferencia que se conviertan en un encuentro entre los diferentes clubes, donde se pueden compartir los mapas creados, realizar lecturas de pasajes y proyectar las fotos y vídeos realizados de los lugares en la actualidad.

\section{LECTORES EN RUTA POR ESPAÑA: RECURSOS LITERARIOS PARA OTROS EVENTOS LETRADOS}

Diversos viajeros escribieron obras de características similares a Viaje por España de Andersen fruto de sus viajes por el país; entre ellos cabe mencionar a Alexander Dumas, que dejó constancia de su visita en 1847 en su obra De París a Cádiz, o Teófilo Gautier, quien describió los lugares de la geografía española en su obra también denominada Viaje por España y publicada en 1840.

A continuación, y con el fin de exponer otros recursos literarios para utilizar en el modelo de club propuesto, "Lectores en ruta", proponemos una lectura para cada una de las provincias de España, si bien en muchos lugares de nuestra geografía podemos encontrarnos con famosas rutas literarias para seguir, marcadas ya con 
itinerarios específicos, como puede ser la Ruta del Quijote en Castilla la Mancha o la Ruta del Hereje en Valladolid; de las cuales se pueden servir también los "Lectores en ruta" -que no podemos obviar pues son bien destacables- y que vienen a enriquecer y a sumar dentro del panorama geográfico literario que proponemos y de los eventos letrados que deriven de las obras literarias. En concreto proponemos las siguientes lecturas, que pueden variar según las preferencias del grupo de lectores:

Álava: La calle de la judería de Toti Martínez de Lezea.

Albacete: Bajo la fría luz de octubre de Eloy M. Cebrián.

Almería: Usura de Pedro Asencio Romero.

Asturias: La Regenta de Leopoldo Alas Clarín.

Ávila: La gloria de Don Ramiro: una vida de tiempos de Felipe II de Enrique Larreta.

Barcelona: Yo fui Johnny Thunders de Carlos Zanón.

Burgos: Inquietud en el paraíso de Oscar Esquivias.

Cáceres: La mediadora de Jesús Sánchez Adalid.

Cádiz: Los aires difíciles de Almudena Grandes.

Cantabria: El camino de Miguel Delibes.

Castellón: Donde nadie te encuentre de Alicia Giménez Barlett.

Ciudad Real: La casa de Juanes de Justo J. García Soriano.

Córdoba: La Feria de los discretos de Pio Baroja.

A Coruña: Memorias de un solterón de Emilia Pardo Bazán.

Ceuta: Te espero en el mar de Diego Canca.

Cuenca: El crimen de Cuenca de Alicio Carcitorial.

Girona: El puente de los judíos de Martí Gironell.

Granada: Cuentos de la Alhambra de Washington Irving.

Guadalajara: El pastor de Flilipa de Luiz Gálvez de Montalvo.

Guipúzcoa: Fuegos con limón de Fernando Aramburu.

Huelva: El rastro de su voz de Antonio j. Sánchez. Huesca: La lluvia amarilla de Julio Llamazares.

Islas Baleares: Za Za, emperador de Ibiza de Ray Loriga.

Jaén: El jinete polaco de Antonio Muñoz Molina.

León: Volverás a Región de Juan Benet.

Lleida: Las voces del pamano de Jaume Cabre i Fabre.
Lugo: Merlin y familia de Álvaro Cunqueiro.

Madrid: Manolito Gafotas de Elvira Lindo.

Málaga: El camino de los ingleses de Antonio Soler.

Melilla: La hija del coronel de Martín Casariego.

Murcia: La carta esférica de Arturo Pérez Reverte.

Navarra: El guardián invisible de Dolores Redondo.

Ourense: La parranda de Eduardo Blanco Amor.

Palencia: El caballero del jabalí blanco de José Javier Esparza.

Pontevedra: Ojos de agua de Domingo Villar.

La Rioja: El baile de los penitentes de Francisco Bescos.

Salamanca: El cuarto de atrás de Carmen Martin Gaite

Santa Cruz de Tenerife: La niebla y la doncella de Lorenzo Silva.

Segovia: Celia, madrecita de Elena Fortún.

Sevilla: Más allá del jardín de Antonio Gala.

Soria: El caballero encantado de Benito Pérez Galdós.

Tarragona: El sicario de los idus de Cristina Teruel. Toledo: Tiempo de arena de Inma Chacón.

Valencia: Entre naranjos de Vicente Blasco Ibáñez.

Valladolid: Capital del dolor de Francisco Umbral. Vizcaya: Días de barrena de Koldo Landaluze.

Zamora: San Manuel Bueno mártir de Miguel de Unamuno.

Zaragoza: Herejía de David Lozano Garbala.

\section{DISCUSIÓN Y CONCLUSIÓN}

Teniendo como punto de partida la definición de un club de lectura y los diferentes tipos de clubes literarios descritos por Calvo (2002), Candamio, (2003), De la Cruz y Saurín-Parra (2005), y Domingo (2007), se ha definido el club de lectura denominado "Lectores en ruta", el cual se convierte en un evento letrado, según la definición de Martos (2010), que conlleva la propuesta de actividades relacionadas con los espacios geográficos de las obras literarias, en consonancia con las propuestas de georrefenciación que describe García (2018), proponiendo el mapeo de las localizaciones y la vinculación de los mismos a los fragmentos de las obras. En lo que se refiere a la propuesta derivada de la obra de Andersen, Viaje por 
España, los clubes organizados y distribuidos por las ciudades por las que transitó el escritor se convertirán en el club anfitrión de su ciudad, encargado de guiar a los clubes visitantes cuando se desarrollen las visitas. En cuanto al viaje, destacar que este puede incluso realizarse de manera virtual, a modo de encuentro online de los diferentes clubes por videoconferencia, donde cada club ofertará una visita virtual al resto.

Para concluir, decir que hemos propuesto un evento letrado en el que la lectura y el viaje van de la mano, convirtiendo el club de lectura -tradicionalmente estático- en un evento en movimiento que bebe de las lecturas propuestas y de los espacios geográficos donde se desarrollan las obras.

La propuesta de club de lectura que proponemos, denominada "Lectores en ruta", enriquece de manera considerable tanto el acto de leer como el acto de viajar, convirtiéndolos en actividades fundamentadas la una en la otra. Y que pretende además convertir la estructura de los clubes de lectura en eventos sociales y colaborativos donde los roles entre participantes y coordinadores se intercambian y democratizan.

\section{BIBLIOGRAFÍA}

ANDERSEN, H. C. (2004). Viaje por España. Madrid: Alianza Editorial.

ARELLANO YANGUAS, V. (1995). "El Club de Lectores: un instrumento para socializar la lectura", en Educación y biblioteca, 61, 57-58.

BARRA HERAS, D., CASTILLO LASALA, M. (2005). "Clubes de lectura y discapacidad", en Mi Biblioteca, 3. Consultado en línea (10/04/2018): https://gredos.usal.es/jspui/ bitstream/10366/119999/1/MB1_N3 P4246.pdf

CALVO, B. (2002). "Recetas para un club de lectura", en Travesía: El portal en internet de las bibliotecas públicas españolas: ideas para bibliotecarios. Consultado en línea (10/04/2018): http://servicios.educarm.es/ templates/portal/ficheros/websDinamicas/154/ recetasparaunclubdelectura.pdf

CANDAMIO GONZÁLEZ, A. (2003). "Los clubes de lectura. Una experiencia de promoción de la lectura", en Educación y biblioteca, 15 (133), 50-51. Consultado en línea (10/04/2018): https://gredos.usal.es/ jspui/bitstream/10366/118896/1/EB15 N133 P50-51.pdf

CORTÉS, J. A. N. (2011). "Clubes de lectura: una oportunidad para El aprendizaje permanente", en A todos/as los/las participantes de La Cátedra Unesco para el "Mejoramiento de la Calidad y Equidad de la Educación en América Latina con base en la Lectura y La Escritura", 59.

DE LA CRUZ, I., SAURIN-PARRA, J. (2005). "Lectores y además amigos: los avatares cotidianos de un club de lectura para adultos", en $\mathrm{Mi}$ Biblioteca, 1(4), 30-35.

DOMINGO ESPINET, G. (2004). "Los clubes de lectura de Barcelona: una experiencia para el fomento de la lectura y del diálogo", en Caro, MartínezConde y Martínez (coord.), La biblioteca pública: compromiso de futuro. Salamanca: Congreso Nacional de Bibliotecas Públicas.

DOMINGO ESPINET, G. (2007). "Leer, conocerse y aprender: la especialización de los clubes de lectura", en Educación y biblioteca, 19 (162), 98-101.

GARCÍA GÓMEZ, S. (2018). "Del texto literario al mapa digital. Las posibilidades de la georreferenciación en los estudios literarios", en Revista de Humanidades Digitales, 2, 120-141.

MARTOS GARCÍA, E. (2010). "Las prácticas de lectura/escritura y los enfoques etnográfico y geográfico", en Didáctica. Lengua y Literatura, 22, 199-229. 